\title{
Determinants of hyperuricemia in non- dialysed chronic kidney disease patients in three hospitals in Cameroon
}

\author{
Marie Doualla ${ }^{1,2^{*}}$ (D), Marie Patrice Halle ${ }^{3}$, Jude Moutchia ${ }^{2}$, Steve Tegang ${ }^{2}$ and Gloria Ashuntantang ${ }^{1}$
}

\begin{abstract}
Background: Chronic kidney disease (CKD) poses a substantial health burden in sub-Saharan Africa, with risk factors ranging from communicable to non-communicable diseases. Hyperuricemia has been recently identified as a factor of progression of CKD. Identifying factors associated with hyperuricemia in CKD patients would help determine interventions to reduce CKD mortality, particularly in resources limited countries. We sought to determine the prevalence and factors associated with hyperuricemia in non-dialysed CKD adult patients in Cameroon.

Methods: This was a cross-sectional study of non-dialysed CKD patients, conducted in 3 referral nephrology units in Cameroon. Relevant clinical and laboratory data were collected using interviewer-administered questionnaires. Serum uric acid, spot urine protein and spot urine creatinine were assessed. Associations between variables were assessed using multivariate analysis. Level of statistical significance was set at $a<0.05$.

Results: A sample of 103 participants was included. Mean age of study participants was $55.78 \pm 12.58$ years, and 59 . $3 \%$ were men. Sixty-nine (67\%) had hyperuricemia. Patient's age (OR: 1.08, 95\% Cl: 1.03-1.13), estimated glomerular filtration rate (OR: $0.94,95 \% \mathrm{Cl}: 0.90-0.98$ ), spot urine protein-creatinine ratio (OR: 1.83, 95\% Cl: 1.07-3.12), no hypertension (OR: 0.09, 95\% Cl: 0.02-0.46), urate lowering therapy (OR: 4.99, 95\% Cl: 1.54-16.16), loop diuretics (OR: 3.39, 95\% Cl: 1.01-11.42), obesity (OR: 6.12, 95\% Cl: 1.15-32.55) and no anaemia (OR: 0.04, 95\% Cl: $0.00-$ 0.29) were independently significantly associated with hyperuricemia.

Conclusions: In this sample of non-dialysed CKD patients in Cameroon, about 7 out of 10 had hyperuricemia. Hyperuricemia was independently associated with patient's age, estimated glomerular filtration rate, spot urine protein-creatinine ratio, hypertension, urate lowering therapy, loop diuretics, obesity and anaemia. More studies are required to establish causal relationships between these associations.
\end{abstract}

Keywords: Chronic kidney disease, Hyperuricemia, Glomerular filtration rate, Proteinuria, Cameroon

\section{Background}

Chronic kidney disease (CKD) poses a substantial health burden in sub-Saharan Africa (SSA), with an estimated prevalence of $13.9 \%$ [1]. Risk factors of CKD in SSA range from communicable to non-communicable diseases $[1,2]$. It is estimated that, by 2030 , more than $70 \%$ of patients with end-stage renal disease (ESRD) will reside in low-income countries, such as countries of SSA [1]. In 2010, out of the 2.6 million people projected worldwide

\footnotetext{
* Correspondence: marie.doualla@gmail.com

${ }^{1}$ Faculty of Medicine and Biomedical Sciences, University of Yaoundé I, Yaoundé, Cameroon

${ }^{2}$ Douala General Hospital, P.O. Box 4856, Douala, Cameroon

Full list of author information is available at the end of the article
}

who accessed renal replacement therapy (RRT), only about $7 \%$ were from low-income and lower-middle income countries $[3,4]$. In Cameroon, the morbidity and mortality of CKD is high, especially amongst patients on haemodialysis [5-7]. Emphasis should therefore be on prevention, early detection and managing factors of progression of CKD, in order to curb incidence of ESRD, while improving access to RRT in SSA [3, 8, 9].

Hyperuricemia has been recently identified as a factor of progression of CKD [10-12]. Findings from some observational studies show that increased serum uric acid is associated with progression of CKD in various subjects [13-18], and is highly associated with ESRD [19-22]. Also, findings

(C) The Author(s). 2018 Open Access This article is distributed under the terms of the Creative Commons Attribution 4.0 International License (http://creativecommons.org/licenses/by/4.0/), which permits unrestricted use, distribution, and 
from interventional studies show that lowering serum uric acid slows down CKD progression [23-25]. Although hyperuricemia is a recognised feature of CKD, studies evaluating its prevalence in CKD are relatively sparse in SSA. Reported prevalence of hyperuricemia in CKD in SSA range from 15.2 to $47.5 \%$ [26, 27].

Hyperuricemia in CKD has been mostly attributed to declining renal function [28]. However, in clinical practice, raised serum uric acid level in CKD is associated with a combination of several other factors, as not every CKD patient presents with hyperuricemia. In non-CKD subjects, factors associated with hyperuricemia include obesity, dyslipidaemia, alcohol consumption, fructose-rich and purine-rich diets, genetics and physical inactivity [29-31]. In CKD patients, factors associated with hyperuricemia have not been fully elucidated.

We therefore sought to determine the prevalence and factors associated with hyperuricemia in non-dialysed adult CKD patients in Cameroon, in order to generate findings that would help determine interventions to reduce CKD mortality, particularly in resources limited countries.

\section{Methods}

\section{Study population and sampling}

This was a cross sectional study carried out in the nephrology units of 3 hospitals in Cameroon (Douala General Hospital [DGH], Yaoundé Central Hospital $[\mathrm{YCH}]$ and Yaoundé University Teaching Hospital [YUTH]) over a period of 6 months (01 February 2014 to 31 July 2014). DGH and YCH are level III (referral) hospitals while YUTH is a level II (secondary) hospital in the Cameroon healthcare system. These sites are referral nephrology centres and receive patients with kidney diseases from all regions of the country.

After prior ethical clearance from the Cameroon $\mathrm{Na}$ tional Ethics Committee, we systematically sampled every non-dialysed CKD patients aged 20 years and above, followed up in the nephrology units of the 3 hospitals. We excluded patients with chronic liver diseases, haemoglobinopathies and/or cancers as reported in their medical charts, and patients who did not give consent.

\section{Data collection}

Socio-demographic data (age, gender) and relevant clinical information (aetiology of CKD, comorbidities, dietary regimens, use of medications known to influence serum uric acid and creatinine levels) were recorded from the patient's medical chart or obtained by interviewer-administered questionnaire. Anthropometric data including height and weight were obtained using a stadiometer (measured to the nearest $0.1 \mathrm{~cm}$, without any foot or head wear) and a manual weighing scale (measured to the nearest $0.1 \mathrm{~kg}$, with light clothing and no foot wear) respectively. Blood pressure was measured according to World Health Organisation (WHO) guidelines [32] using an automatic blood pressure machine $\left(\mathrm{OMRON}^{\circ}\right.$ M2, HEM-7121-E). The most recent (less than 3 months) values of haemoglobin, serum high-density lipoprotein cholesterol (HDL-c), serum low-density lipoprotein cholesterol (LDL-c), serum total cholesterol, serum triglycerides and plasma creatinine in patient's medical charts were recorded.

Uric acid in the serum specimen was measured using the direct kinetic uricase method [33]. A clean-catch mid-stream urine specimen from all participants was collected for spot protein and creatinine assessment using the Benzethonium Chloride [34] and Jaffé method [35] respectively.

\section{Definitions and calculations}

Estimated glomerular filtration rate (eGFR) was computed from serum creatinine using the Chronic Kidney Disease Epidemiology Collaboration (CKD-EPI) equation [36]. CKD was defined by eGFR $<60 \mathrm{ml} / \mathrm{min}$ per $1.73 \mathrm{~m}^{2}$ for more than 3 months [37]. CKD was classified according to Kidney Disease: Improving Global Outcomes (KDIGO guidelines) into Stage 1/G1 (eGFR $\geq 90 \mathrm{ml} / \mathrm{min}$ per $\left.1.73 \mathrm{~m}^{2}\right)$, Stage $2 / \mathrm{G} 2 \quad($ eGFR $=60$ $89 \mathrm{ml} / \mathrm{min}$ per $1.73 \mathrm{~m}^{2}$ ), Stage $3 / \mathrm{G} 3 \mathrm{a}(\mathrm{eGFR}=45-59 \mathrm{ml} /$ min per $\left.1.73 \mathrm{~m}^{2}\right)$, Stage $3 \mathrm{~b} / \mathrm{G} 3 \mathrm{~b}(\mathrm{eGFR}=30-44 \mathrm{ml} / \mathrm{min}$ per $\left.1.73 \mathrm{~m}^{2}\right)$, Stage $4 / \mathrm{G} 4(\mathrm{eGFR}=15-29 \mathrm{ml} / \mathrm{min}$ per $\left.1.73 \mathrm{~m}^{2}\right)$, Stage $5 /$ G5 $\left(\right.$ eGFR $<15 \mathrm{ml} / \mathrm{min}$ per $\left.1.73 \mathrm{~m}^{2}\right)$ [37]. Proteinuria was classified into normal or mild increase/A1 (spot urine protein-creatinine ratio $[\mathrm{PCR}]<$ $150 \mathrm{mg} / \mathrm{g}$ ), moderate increase $/ \mathrm{A} 2$ (spot urine $\mathrm{PCR}=$ $150-500 \mathrm{mg} / \mathrm{g}$ ) and severe increase/A3 (spot urine PCR $>500 \mathrm{mg} / \mathrm{g}$ ) [37].

Renal disease was Glomerular if manifesting with glomerular range proteinuria, and/or haematuria (deformed or red blood cells cast), associated with hypertension and/or oedema; Tubulo-interstitial if manifesting with sterile leucocyturia, and low urine specific gravity, associated with or without non-glomerular haematuria and proteinuria; Vascular if presence of hypertension, with moderate proteinuria and normal urine sediment. Mixed renal involvement was renal disease manifesting with a combination of 2 or more of glomerular, tubulo-interstitial and vascular involvement.

Hyperuricemia was defined as serum uric acid > $70 \mathrm{mg} / \mathrm{l}$ in males and $>60 \mathrm{mg} / \mathrm{l}$ in females [38]. Hypertension was defined as a systolic blood pressure $\geq$ $140 \mathrm{mmHg}$ and/or a diastolic blood pressure $\geq 90$ or current use of antihypertensive drugs. Diabetes was defined as history of diabetes confirmed in the patient's medical records and/or current use of blood glucose control medications. Gout was defined as history of gouty arthritis confirmed in the patient's medical records and/or current use of urate lowering therapy (ULT) against gout. Low protein, low-carb and low fat diet 
were defined as a dietary regimen poor in proteins (less than $30 \mathrm{~g}$ daily), sugars and lipids respectively, as prescribed by a dietician. Body mass index (BMI) was classified as; underweight $\left(\mathrm{BMI}<18.5 \mathrm{~kg} / \mathrm{m}^{2}\right)$, normal weight $\left(\mathrm{BMI}=18.5-24.9 \quad \mathrm{~kg} / \mathrm{m}^{2}\right), \quad$ overweight $\quad(\mathrm{BMI}=25-$ $\left.29.9 \mathrm{~kg} / \mathrm{m}^{2}\right)$ and obesity $\left(\mathrm{BMI} \geq 30 \mathrm{~kg} / \mathrm{m}^{2}\right)$ [39]. Anaemia was defined as haemoglobin $<13.0 \mathrm{~g} / \mathrm{dl}$ in males and $<$ $12.0 \mathrm{~g} / \mathrm{dl}$ in females [40]. Dyslipidaemia was defined as total cholesterol $\geq 240 \mathrm{mg} / \mathrm{dl} \quad(6.21 \mathrm{mmol} / \mathrm{l})$ or low-density lipoprotein cholesterol (LDL-c) $>160 \mathrm{mg} / \mathrm{dl}$ $(4.14 \mathrm{mmol} / \mathrm{l})$ or high-density lipoprotein cholesterol $(\mathrm{HDL}-\mathrm{c})<40 \mathrm{mg} / \mathrm{dl} \quad(1.03 \mathrm{mmol} / \mathrm{l})$ or triglycerides $\geq 150 \mathrm{mg} / \mathrm{dl}(1.69 \mathrm{mmol} / \mathrm{l})$ [41].

\section{Statistical analysis}

Statistical analyses were done using Statistical Package for Social Sciences (SPSS), version 23 Inc., Chicago, Illinois, USA). Normality of continuous data was assessed using Shapiro-Wilk test. Continuous variables were summarised as means and standard deviations (SD), and medians and interquartile ranges (IQR) where appropriate. Categorical variables were summarised as frequencies and percentages.

One-Way ANOVA was used to compare means between more than 2 independent groups. Bivariate analyses were done using generalized linear models (using serum uric acid as outcome variable) and logistic regression (using hyperuricemia as outcome variable). After bivariate analyses, variables with $p<0.02$ were included as adjustment variables in multivariate analyses. Continuous data were selected over categorical data for variables with both continuous and categorical data. In order to avoid multicollinearity, bivariate correlations were assessed between the predictor variables prior to multivariate analyses. Predictor variables which were highly significantly $(p<0.01)$ on bivariate correlation were excluded from the multivariate analysis. Furthermore, collinearity diagnostics were done to ensure that none of the predictor variables had Tolerance $<0.3$ and/or Variance Inflation Factor $>3$. There were missing data in lipid profile indices (Total Cholesterol [10 missing], HDL-c [11 missing], LDL-c [11 missing], and Triglycerides [14 missing]. Missing data where excluded from the analysis pairwise. Level of statistical significance was set at $\alpha<0.05$.

Sensitivity analyses were done, defining hyperuricemia as serum uric acid $>70 \mathrm{mg} / \mathrm{l}$ in males and $>60 \mathrm{mg} / \mathrm{l}$ in females or current use of ULT. The results were very similar to our current results.

\section{Results}

Out of the 198 non-dialysed CKD patients aged 20 years and above who attended our study sites during the study period, 2 patients were excluded for chronic liver disease (liver cirrhosis), one patient excluded for haemoglobinopathy (sickle cell disease), and one patient excluded for cancer (prostate cancer). Of the 194 eligible CKD patients approached for participation in the study, 103 patients gave consent and were included into study; thus a participation rate of $53.1 \%$.

\section{Baseline characteristics of study participants}

Mean age of our study participants was $55.78 \pm$ 12.58 years, and 61 (59.3\%) were males. The median (IQR) eGFR was $17.00(11.00) \mathrm{ml} / \mathrm{min}$ per $1.73 \mathrm{~m}^{2}$, and median (IQR) spot urine PCR ratio was 803.0 (1120.0) $\mathrm{mg} / \mathrm{g}$. More than two-thirds of participants were at an advanced stage of CKD with $48 \%$ at stage 4 and $25.2 \%$ at stage 5 . The most common comorbidities identified were hypertension (87.4\%), diabetes (34.0\%) and gout (21.4\%). The types of renal involvement included; glomerular (60.2\%), vascular (19.4\%), tubulo-insterstitial (11.7\%), and mixed (8.7\%). Forty-two (40.2\%) participants were on ULT (Allopurinol), 32 (31.1\%) were on loop diuretics (Furosemide), while 5 (4.9\%) were on angiotensin receptor blockers (Losartan). The mean serum uric acid of the study participants was $76.03 \pm 20.50 \mathrm{mg} / \mathrm{l}$. Table 1 .

\section{Prevalence of hyperuricemia in chronic kidney disease}

The prevalence of hyperuricemia amongst our study participants was $67 \%$ (95\% CI: 58.3-75.7\%).

\section{Factors associated with hyperuricemia in chronic kidney disease}

On bivariate analysis, patient's age ( $\beta$ : $0.54,95 \% \mathrm{CI}$ : $0.25-0.84$ ), eGFR ( $\beta$ : $-0.67,95 \%$ CI: $-0.91--0.43)$, CKD stage 4 ( $\beta$ : $20.55,95 \%$ CI: $10.32-30.76)$ or CKD stage 5 ( $\beta$ : 20.82, 95\% CI: 9.55-32.09), spot urine PCR ( $\beta$ : 3.76, 95\% CI: $1.12-6.40)$, severe proteinuria ( $\beta$ : $16.51,95 \% \mathrm{CI}$ : 6.56-26.46), no hypertension ( $\beta$ : $-17.83,95 \%$ CI: -27.17 -8.48 ), systolic ( $\beta$ : $0.25,95 \%$ CI: $0.09-0.40)$ and diastolic ( $\beta$ : $0.58,95 \%$ CI: $0.14-1.01)$ blood pressures, diabetes $(\beta$ : 9.01, 95\% CI: $0.87-17.14$ ), low protein diet ( $\beta$ : $-8.31,95 \%$ CI: -16.07 - -0.55), low-Carb diet ( $\beta$ : 8.36 , 95\% CI: $0.64-$ 16.07), ULT ( $\beta$ : 22.71, 95\% CI: $16.00-29-42$ ), loop diuretics ( $\beta$ : $11.94,95 \%$ CI: 3.75-20.13), body mass index $(\beta: 1.06,95 \%$ CI: $0.07-2.05)$ and no anaemia $(\beta:-27.42$, 95\% CI: $(-39.02-15.82)$ were significantly associated with serum uric acid. Mean serum uric acid significantly differed across CKD stages (F: 7.91, $p$ value $<0.001$ ) Fig. 1, and significantly differed by severity of proteinuria (F: $5.46, p$ value $=0.006$ ) Fig. 2.

Also, on bivariate logistic regression, patient's age (OR: 1.05, 95\% CI: 1.02-1.09), eGFR (OR: 0.93, 95\% CI: 0.890.96), CKD stage 4 (OR: 9.11, 95\% CI: 2.01-33.21) or CKD stage 5 (OR: 6.67, 95\% CI: 1.63-27.27), spot urine PCR (OR: 1.52, 95\% CI: 1.01-2.30), severe proteinuria (OR: 4.71, 95\% CI: 1.59-13.99), no hypertension (OR: 
Table 1 Baseline characteristics of study participants

\begin{tabular}{|c|c|}
\hline Variable & Total $(N=103)$ \\
\hline Age (years), mean $\pm S D$ & $55.78 \pm 12.58$ \\
\hline \multicolumn{2}{|l|}{ Age Strata, n (\%) } \\
\hline $20-29$ years & $2(1.9)$ \\
\hline 30-39 years & $9(8.7)$ \\
\hline $40-49$ years & 19 (18.4) \\
\hline $50-59$ years & $33(32.0)$ \\
\hline $60-69$ years & $23(22.3)$ \\
\hline$>70$ years & $17(16.5)$ \\
\hline Male gender, n (\%) & $61(59.2)$ \\
\hline Duration of CKD diagnosis (months), mean \pm SD & $14.67 \pm 18.05$ \\
\hline \multicolumn{2}{|l|}{ CKD stage, n (\%) } \\
\hline Stage $1 / \mathrm{G} 1$ & $0(0.0)$ \\
\hline Stage $2 / G 2$ & $4(3.9)$ \\
\hline Stage $3 a / G 3 a$ & $8(7.8)$ \\
\hline Stage 3b/G3b & $15(14.6)$ \\
\hline Stage $4 / G 4$ & $50(48.5)$ \\
\hline Stage $5 / G 5$ & $26(25.2)$ \\
\hline \multicolumn{2}{|l|}{ Type of renal involvement, n (\%) } \\
\hline Glomerular & $62(60.2)$ \\
\hline Tubulo-interstitial & $12(11.7)$ \\
\hline Vascular & $20(19.4)$ \\
\hline Mixed & $9(8.7)$ \\
\hline \multicolumn{2}{|l|}{ Comorbidities, n (\%) } \\
\hline Hypertension & $90(87.4)$ \\
\hline Diabetes & $35(34.0)$ \\
\hline Gout & $22(21.4)$ \\
\hline HIV & $13(12.6)$ \\
\hline \multicolumn{2}{|l|}{ Dietary regimen, $\mathrm{n}(\%)$} \\
\hline None & $32(31.1)$ \\
\hline Low protein & $46(55.3)$ \\
\hline Low-carb & $52(50.5)$ \\
\hline Low fat & $29(28.2)$ \\
\hline \multicolumn{2}{|l|}{ Medications, n (\%) } \\
\hline aUrate lowering therapy & $42(40.8)$ \\
\hline bLoop diuretics & $32(31.1)$ \\
\hline${ }^{\mathrm{C}}$ Thiazide diuretics & $1(1.0)$ \\
\hline${ }^{d} A R B$ & $5(4.9)$ \\
\hline Systolic Blood Pressure $(\mathrm{mmHg})$, mean \pm SD & $142.3 \pm 30.3$ \\
\hline Diastolic Blood Pressure $(\mathrm{mmHg})$, mean \pm SD & $88.3 \pm 21.3$ \\
\hline Weight (kg), median (IQR) & $75.00(18.40)$ \\
\hline Height (m), median (IQR) & $1.66(0.10)$ \\
\hline BMI $\left(\mathrm{kg} / \mathrm{m}^{2}\right)$, median (IQR) & $26.30(6.04)$ \\
\hline Haemoglobin $(\mathrm{g} / \mathrm{dl})$, mean \pm SD & $10.27 \pm 1.76$ \\
\hline Total Cholesterol $(\mathrm{mmol} / \mathrm{l})$, mean $\pm \mathrm{SD}$ & $2.06 \pm 0.56$ \\
\hline
\end{tabular}

Table 1 Baseline characteristics of study participants (Continued)

\begin{tabular}{|c|c|}
\hline Variable & Total $(N=103)$ \\
\hline $\mathrm{HDL}-\mathrm{c}(\mathrm{mmol} / \mathrm{l})$, mean $\pm \mathrm{SD}$ & $0.55 \pm 0.19$ \\
\hline LDL-c (mmol/l), mean \pm SD & $1.25 \pm 0.42$ \\
\hline Tryglycerides (mmol/l), median (IQR) & $1.04(0.72)$ \\
\hline Plasma creat (mg/dl), median (IQR) & $3.65(1.55)$ \\
\hline eGFR $\left(\mathrm{ml} / \mathrm{min}\right.$ per $\left.1.73 \mathrm{~m}^{2}\right)$, median (IQR) & $17.00(11.00)$ \\
\hline Spot urine protein $(\mathrm{mg} / \mathrm{dl})$, median (IQR) & $1.07(1.06)$ \\
\hline Spot urine creat (mg/dl), median (IQR) & $1.47(1.14)$ \\
\hline Spot urine PCR (mg/g), median (IQR) & $803.0(1120.0)$ \\
\hline Serum uric acid $(\mathrm{mg} / \mathrm{l})$, mean $\pm \mathrm{SD}$ & $76.19 \pm 20.25$ \\
\hline Hyperuricemia, n (\%) & $69(67.0)$ \\
\hline \multicolumn{2}{|c|}{$\begin{array}{l}\text { CKD Chronic kidney disease, HIV Human immuno-deficiency virus, } A R B \\
\text { Angiotensin receptor blockers, BMI Body mass index, } H D L \text {-c high-density } \\
\text { lipoprotein cholesterol, } L D L-c \text { low-density lipoprotein cholesterol, Creat } \\
\text { Creatinine, eGFR Estimated glomerular filtration rate, } P C R \text { Protein-to-creatinine } \\
\text { ratio, } S D \text { standard deviation, IQR interquartile range } \\
\text { aAllopurinol } \\
\text { bFurosemide } \\
\text { cHydrochlorothiazide } \\
\text { dLosartan }\end{array}$} \\
\hline
\end{tabular}

0.06, 95\% CI: 0.01-0.30), systolic (OR: 1.03, 95\% CI: 1.01-1.06) and diastolic (OR: 1.05, 95\% CI: 1.00-1.11) blood pressures, low protein diet (OR: 0.42 , 95\% CI: 0.18-0.98), low fat diet (OR: 3.09, 95\% CI: 1.06-9.02), ULT (OR: 6.71, 95\% CI: 2.32-19.37), loop diuretics (OR: 3.73, 95\% CI: 1.29-10.82), obesity (OR: 6.65, 95\% CI: 1.36-32.61) and no anaemia (OR: 0.04, 95\% CI: 0.00 0.29 ) were significantly associated with hyperuricemia.

On multivariate analysis, after adjusting for patient's age, eGFR, spot urine PCR, systolic blood pressure, ULT, loop diuretics and haemoglobin level, patient's age ( $\beta$ : 0.49, 95\% CI: $0.24-0.74)$, eGFR ( $\beta$ : -0.36 , 95\% CI: $-0.57--0.16)$, CKD stage 4 ( $\beta$ : $17.49,95 \%$ CI: 9.52-25.45) or CKD stage 5 ( $\beta$ : 13.28, 95\% CI: 4.41-22.16), spot urine PCR ( $\beta$ : 3.06 , 95\% CI: $1.09-5.04)$, severe proteinuria ( $\beta$ : $13.94,95 \%$ CI: $4.92-$ $22.95)$, no hypertension ( $\beta$ : $-18.37,95 \%$ CI: -28.67 - -8.06), systolic ( $\beta$ : 0.14, 95\% CI: 0.02-0.25) and diastolic ( $\beta$ : 0.35, 95\% CI: 0.03-0.66) blood pressures, ULT ( $\beta: 18.84$, 95\% CI: 12.98-24.71), loop diuretics ( $\beta$ : 6.62, 95\% CI: 0.25-12.99) and no anaemia ( $\beta$ : $-18.08,95 \%$ CI: $(-27.55--8.61)$ were significantly associated with serum uric acid. Table 2.

On multivariate logistic regression, after adjusting for patient's age, eGFR, spot urine PCR, systolic blood pressure, ULT, loop diuretics and haemoglobin level, patient's age (OR: 1.08, 95\% CI: 1.03-1.13), GFR (OR: 0.94, 95\% CI: 0.90-0.98), CKD stage 4 (OR: 24.66, 95\% CI: 3.63-167.63) or CKD stage 5 (OR: 7.73, 95\% CI: $1.63-$ 27.27), spot urine PCR (OR: 1.83, 95\% CI: 1.07-3.12), severe proteinuria (OR: 6.27, 95\% CI: 1.66-23.60), no hypertension (OR: 0.09, 95\% CI: 0.02-0.46), systolic blood pressure (OR: 1.04, 95\% CI: 1.01-1.07), ULT (OR: 4.99, 95\% CI: 1.54-16.16), loop diuretics (OR: 3.39, 95\% CI: 1.01-11.42), obesity (OR: 6.12, 95\% CI: 1.15-32.55) 


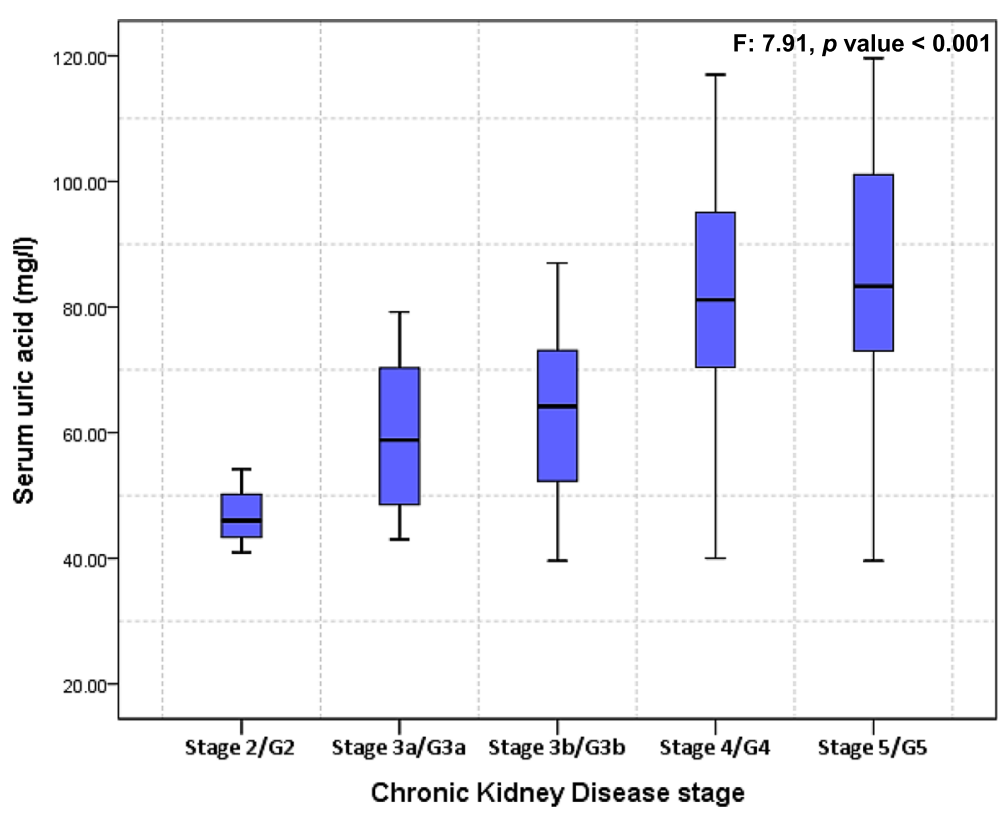

Fig. 1 Boxplot of Serum uric acid versus Chronic Kidney Stage

and no anaemia (OR: 0.04, 95\% CI: 0.00-0.46) were significantly associated with hyperuricemia. Table 2.

\section{Discussion}

Our study showed that more than half of CKD patients had hyperuricemia. Among a wide range of demographic, clinical and laboratory parameters evaluated, patient's age, eGFR, spot urine PCR, hypertension, ULT, loop diuretics and anaemia were significantly associated with serum uric acid and hyperuricemia in CKD.
Hyperuricemia is common in CKD and mostly results from decreased uric acid excretion [10]. The kidneys play a major role in excreting uric acid, an end-product of purine metabolism [28]. We found a higher prevalence of hyperuricemia in CKD in our setting, compared to other SSA settings such as Nigeria [26] and Chad [27]. These divergent results can be largely attributed to study methodology. In the Nigerian study, the authors evaluated 120 pre-dialysis CKD patients and reported a prevalence of $47.5 \%$. However, they excluded patients

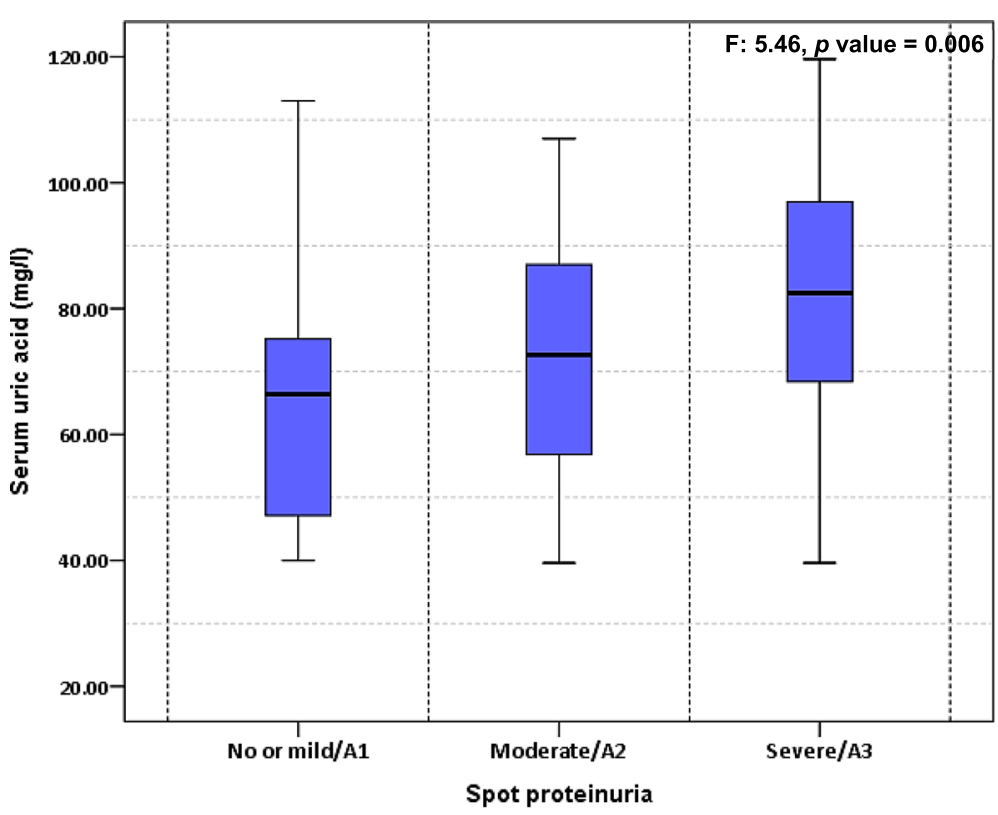

Fig. 2 Box plot of Serum uric acid versus stage of proteinuria 
Table 2 Factors associated with serum uric acid and hyperuricemia in Chronic Kidney Disease

\begin{tabular}{|c|c|c|c|c|}
\hline \multirow[t]{3}{*}{ Variable } & \multicolumn{2}{|l|}{ Serum uric acid } & \multicolumn{2}{|l|}{ Hyperuricemia } \\
\hline & Bivariate analysis & ${ }^{a}$ Multivariate analysis & Bivariate analysis & ${ }^{a}$ Multivariate analysis \\
\hline & $\beta(95 \% \mathrm{Cl})$ & $\beta(95 \% \mathrm{Cl})$ & cOR $(95 \% \mathrm{Cl})$ & aOR $(95 \% \mathrm{Cl})$ \\
\hline Age & $0.54(0.25-0.84)$ & $0.49(0.24-0.74)$ & $1.05(1.02-1.09)$ & $1.08(1.03-1.13)$ \\
\hline Male gender & $0.15(-1.79-14.41)$ & $1.96(-4.41-8.34)$ & $0.85(0.37-1.98)$ & $0.58(0.17-1.91)$ \\
\hline Duration of CKD diagnosis (months) & $-0.01(-0.23-0.22)$ & $-0.11(-0.26-0.05)$ & $1.01(0.99-1.04)$ & $0.99(0.96-1.03)$ \\
\hline GFR (ml/min per $\left.1.73 \mathrm{~m}^{2}\right)$ & $-0.67(-0.91--0.43)$ & $-0.36(-0.57--0.16)$ & $0.93(0.89-0.96)$ & $0.94(0.90-0.98)$ \\
\hline \multicolumn{5}{|l|}{ CKD stage } \\
\hline Stage $2 / G 2$ & $-15.08(-34.64-4.48)$ & $2.68(-17.85-12.49)$ & - & - \\
\hline Stage 3a/G3a & $3.61(-11.61-18.83)$ & $10.79(-1.16-22.75)$ & $1.2(0.20-7.18)$ & $5.44(0.45-65.66)$ \\
\hline Stage 3b/G3b & Ref. & Ref. & Ref. & Ref. \\
\hline Stage $4 / G 4$ & $20.55(10.32-30.76)$ & $17.49(9.52-25.45)$ & $9.11(2.01-33.21)$ & $24.66(3.63-167.63)$ \\
\hline Stage $5 / G 5$ & $20.82(9.55-32.09)$ & $13.28(4.41-22.16)$ & $6.67(1.63-27.27)$ & $7.73(1.06-56.30)$ \\
\hline Spot urine PCR (mg/g) & $3.76(1.12-6.40)$ & $3.06(1.09-5.04)$ & $1.52(1.01-2.30)$ & $1.83(1.07-3.12)$ \\
\hline \multicolumn{5}{|l|}{ Proteinuria } \\
\hline No or Mild/A1 & Ref. & Ref. & Ref. & Ref. \\
\hline Moderate/A2 & $8.81(-3.08-20.70)$ & $5.41(-5.26-16.07)$ & $1.99(0.57-6.90)$ & $1.65(0.39-6.90)$ \\
\hline Severe/A3 & $16.51(6.56-26.46)$ & $13.94(4.92-22.95)$ & $4.71(1.59-13.99)$ & $6.27(1.66-23.60)$ \\
\hline \multicolumn{5}{|l|}{ Type of renal involvement } \\
\hline Glomerular & Ref. & Ref. & Ref. & Ref. \\
\hline Tubulointerstitial & $-3.31(-15.63-9.02)$ & $5.76(-3.47-14.99)$ & $0.77(0.22-2.72)$ & $1.71(0.30-9.67)$ \\
\hline Vascular & $7.36(-2.69-17.41)$ & $4.39(-2.97-11.74)$ & $1.10(0.25-4.83)$ & $2.25(0.43-11.71)$ \\
\hline Mixed & $-8.92(-22.86-5.02)$ & $-5.67(-15.70-4.36)$ & $2.20(0.65-7.40)$ & $1.57(0.24-10.19)$ \\
\hline No Hypertension & $-17.83(-27.17--8.48)$ & $-18.37(-28.67--8.06)$ & $0.06(0.01-0.30)$ & $0.09(0.02-0.46)$ \\
\hline Systolic Blood Pressure (mmHg) & $0.25(0.09-0.40)$ & $0.14(0.02-0.25)$ & $1.03(1.01-1.06)$ & $1.04(1.01-1.07)$ \\
\hline Diastolic Blood Pressure (mmHg) & $0.58(0.14-1.01)$ & $0.35(0.03-0.66)$ & $1.05(1.00-1.11)$ & $1.06(0.99-1.13)$ \\
\hline Diabetes & $9.01(0.87-17.14)$ & $4.34(-2.72-11.40)$ & $2.09(0.83-5.29)$ & $1.40(0.47-4.18)$ \\
\hline HIV & $-8.70(-20.45-3.04)$ & $-5.15(-13.87-3.57)$ & $0.76(0.23-2.53)$ & $1.37(0.27-6.99)$ \\
\hline Low protein diet & $-8.31(-16.07--0.55)$ & $-1.45(-7.70-4.80)$ & $0.42(0.18-0.98)$ & $0.80(0.29-2.21)$ \\
\hline Low-Carb diet & $8.36(0.64-16.07)$ & $-0.67(-7.18-5.84)$ & $2.10(0.91-4.86)$ & $1.09(0.41-2.91)$ \\
\hline Low fat diet & $6.20(-2.47-14.88)$ & $-0.92(-7.16-5.32)$ & $3.09(1.06-9.02)$ & $1.73(0.44-6.81)$ \\
\hline bUrate lowering therapy & $22.71(16.00-29-42)$ & $18.84(12.98-24.71)$ & $6.71(2.32-19.37)$ & $4.99(1.54-16.16)$ \\
\hline${ }^{\complement}$ Loop diuretics & $11.94(3.75-20.13)$ & $6.62(0.25-12.99)$ & $3.73(1.29-10.82)$ & $3.39(1.01-11.42)$ \\
\hline${ }^{\mathrm{d}}$ Thiazide diuretics & $4.82(-35.34-44.98)$ & $-6.43(-34.72-21.86)$ & - & - \\
\hline${ }^{\mathrm{e}} \mathrm{ARB}$ & $-1.46(-19.78-16.87)$ & $1.54(11.69-14.77)$ & $0.73(0.12-4.57)$ & $2.38(0.08-69.14)$ \\
\hline BMI $\left(\mathrm{kg} / \mathrm{m}^{2}\right)$ & $1.06(0.07-2.05)$ & $0.49(-0.35-1.32)$ & $1.12(0.99-1.26)$ & $1.04(0.89-1.21)$ \\
\hline \multicolumn{5}{|l|}{ BMI class } \\
\hline Normal & Ref. & Ref. & Ref. & Ref. \\
\hline Overweight & $4.99(-3.61-13.59)$ & $5.52(-1.38-12.41)$ & $1.38(0.57-3.35)$ & $1.59(0.56-4.53)$ \\
\hline Obesity & $11.29(0.57-22.02)$ & $4.34(-4.48-13.16)$ & $6.65(1.36-32.61)$ & $6.12(1.15-32.55)$ \\
\hline Haemoglobin (g/dl) & $-2.82(-5.00--0.63)$ & $-1.61(-3.26-0.04)$ & $0.77(0.61-0.99)$ & $0.86(0.64-1.15)$ \\
\hline No anaemia & $-27.42(-39.02--15.82)$ & $-18.08(-27.55--8.61)$ & $0.04(0.00-0.29)$ & $0.04(0.00-0.46)$ \\
\hline Total Cholesterol (mmol/l) & $4.85(-8.33-18.0)$ & $9.24(-2.54-21.02)$ & $1.64(0.38-7.40)$ & $1.05(0.11-9.77)$ \\
\hline HDL-c (mmol/l) & $-29.28(-67.02-8.46)$ & $-4.16(-33.53-25.22)$ & $0.11(0.01-7.30)$ & $0.01(0.00-30.34)$ \\
\hline LDL-c (mmol/l) & $4.81(-12.63-22.25)$ & $-1.06(-14.88-12.76)$ & $1.81(0.24-13.82)$ & $0.38(0.21-6.91)$ \\
\hline
\end{tabular}


Table 2 Factors associated with serum uric acid and hyperuricemia in Chronic Kidney Disease (Continued)

\begin{tabular}{|c|c|c|c|c|}
\hline \multirow[t]{3}{*}{ Variable } & \multicolumn{2}{|l|}{ Serum uric acid } & \multicolumn{2}{|l|}{ Hyperuricemia } \\
\hline & Bivariate analysis & aMultivariate analysis & Bivariate analysis & ${ }^{a}$ Multivariate analysis \\
\hline & $\beta(95 \% \mathrm{Cl})$ & $\beta(95 \% \mathrm{Cl})$ & cOR $(95 \% \mathrm{Cl})$ & $\mathrm{aOR}(95 \% \mathrm{Cl})$ \\
\hline Triglycerides (mmol/l) & $5.30(-7.67-18.27)$ & $3.76(-5.59-13.11)$ & $1.34(0.32-5.67)$ & $1.40(0.96-12.34)$ \\
\hline Dyslipidaemia & $10.62(-4.60-25.85)$ & $11.23(-1.58-24.03)$ & $4.25(0.82-22.13)$ & $2.13(0.13-34.26)$ \\
\hline
\end{tabular}

with gouty arthritis and patients on ULT. In the Chadian study, after evaluating 712 CKD patients, an even lower prevalence of $15.2 \%$ was reported. However, in this Chadian study, no information was provided on whether patients were on maintenance dialysis or not. Our higher prevalence could also be due to the high proportion of advanced CKD (73.2\% CKD stages 4 and 5) amongst our study participants. Our prevalence was similar to the $70 \%$ reported in a paediatric population of CKD patients in USA [42]. The high proportion of advanced CKD amongst our study participants is due to the fact that our study was conducted in referral nephrology units which receive advanced cases of CKD from peripheral nephrology units.

We found significant association between eGFR, spot urine PCR and serum uric acid. Patients with CKD stages 4 or 5 and had 24.66 and 7.73 times higher significant odds respectively, of having hyperuricemia compared to those with CKD stage 3b. Also, patients with severe proteinuria had 6.27 significant higher odds of having hyperuricemia compared to those with no or mild proteinuria. These findings were similar to the results of Krishnan [43]. We also found a significant association between loop diuretics and serum uric acid in our sample of non-dialysed CKD. It is known that, loop diuretics predisposes individuals to hyperuricemia [44]. Diuretics cause hyperuricemia by increasing urate absorption and decreasing urate secretion in the kidneys [44]. Loop diuretics used in the management of hypertension and fluid retention in CKD should therefore be used judiciously.

We found a significant independent association between hypertension and hyperuricemia. Noone et al.. also reported a significant association between hypertension and hyperuricemia in paediatric patients with CKD [42]. A growing body of evidence suggest uric acid levels play a role in the development of hypertension $[45,46]$. Uric acid can cause hypertension by mediating pro inflammatory pathways in vascular smooth muscles, inhibition of endothelial nitric oxide and activation of the renin-angiotensin system $[45,46]$. On the other hand, hypertension increases renal vascular resistance, reducing renal flow, those increasing urate reabsorption [30]. Also, use of antihypertensive drugs like diuretics can increase serum uric acid levels [44]. It is however unlikely that in our study, the observed association between hypertension and hyperuricemia is due to use of diuretics as this was adjusted for in our analysis. Sedaghat et al. suggested that hypertension mediates the decline in renal function caused by hyperuricemia [47]. Further research is needed to clarify the relationship between hyperuricemia and hypertension in CKD patients.

Subjects who were obese had 6.12 times significant higher odds of having hyperuricemia. This was in accordance with findings by Noone et al, in their paediatric CKD population [42]. Hyperuricemia seen in obesity has been attributed to insulin resistance and higher leptin production [30]. Insulin resistance in obese individuals causes larger amounts of insulin to be secreted in order to maintain glucose metabolism. The kidney responds to this hyperinsulinemia by decreasing urate clearance [48]. Obesity may therefore be a cause of hyperuricemia in our subjects, but a causal relationship between these two variables was not evaluated in this study.

It is possible that the observed association between hyperuricemia and anaemia is just an incidental finding. Both conditions can be caused by the underlying CKD, and there may not be a direct relationship between the two. However, in our analysis, after adjusting for eGFR and spot urine PCR, we still found a significant association between both variables. CKD patients with no anaemia displayed 0.04 times lower odds of having hyperuricemia after relevant confounder adjustment. To the best of our knowledge, there has been no study to depict the relationship between hyperuricemia and anaemia in CKD. In the Artherosclerosis Risk in the Communities (ARIC) study, McAdams-DeMarco et al. [49], after following up 10,791 individuals for a period of 9 years, reported that anaemia was associated with an approximately two-fold increased risk of 'self-reported' 
gout independent of renal function. The authors had no clear biological explanation for the link between anaemia and hyperuricemia, but hypothesised that this link may be mediated by oxidative stress. Increased oxidative stress seen in anaemia could cause hyperuricemia by increasing xanthine oxidase activity and increased cell death/turnover [50]. More research is needed to explicate this relationship.

Our study had strengths and limitations. It was well designed to capture a wide range of demographic, clinical and biological variables that could influence hyperuricemia in CKD. Furthermore, important variables such as medications and diet that could influence our findings were taken into consideration and accounted for. However the small sample size may have limited statistical power of some analyses. Also, odds ratios on logistic regression overestimate relative risks when the prevalence is high. In addition, the cross-sectional design did not enable us carryout cause and effect analysis for the various associations seen. This was particularly true for the associations seen between hypertension, obesity and anaemia with hyperuricemia.

\section{Conclusions}

In this sample of non-dialysed CKD followed-up in referral centres in Cameroon, about 7 out of 10 had hyperuricemia. Hyperuricemia was independently associated with patient's age, eGFR, spot urine PCR, hypertension, ULT, loop diuretics, obesity and anaemia. More studies are required to establish causal relationships between these associations.

\section{Abbreviations}

BMI: Body mass index; CKD: Chronic kidney disease; DGH: Douala General Hospital; eGFR: Estimated glomerular filtration rate; ESRD: End-stage renal disease; HDL-C: Serum high-density lipoprotein cholesterol; IIQR: Interquartile ranges; LDL-C: Serum low-density lipoprotein cholesterol; PCR: Spot urine protein-creatinine ratio; RRT: Renal replacement therapy; SD: Standard deviation; SSA: Sub-Saharan Africa; ULT: Urate lowering therapy;

YCH: Yaoundé Central Hospital; YUTH: Yaoundé University Teaching Hospital

\section{Acknowledgements}

We thank the staff at the Rheumatology Unit for their cooperation during this study and especially the study participants for accepting to take part in the study.

\section{Availability of data and materials}

The datasets used and/or analysed during the current study are available from the corresponding author on reasonable request.

\section{Authors' contributions \\ Study conception and design: MD, GA, MPH. Data collection: MD, ST. Statistical analysis: JM. Drafting of manuscript: ST, JM, MD, MPH, GA. Critical review of manuscript: $\mathrm{MD}, \mathrm{MPH}$. All authors read and approved the final version of the manuscript.}

\section{Ethics approval and consent to participate}

We obtained ethical clearance from the Cameroon National Ethics Committee. Written consent was obtained from all patients to participate to All participants gave their consent to this study.
Consent for publication

Not applicable.

\section{Competing interests}

The authors declare that they have no competing interests.

\section{Publisher's Note}

Springer Nature remains neutral with regard to jurisdictional claims in published maps and institutional affiliations.

\section{Author details}

${ }^{1}$ Faculty of Medicine and Biomedical Sciences, University of Yaoundé I, Yaoundé, Cameroon. ²Douala General Hospital, P.O. Box 4856, Douala, Cameroon. ${ }^{3}$ Faculty of Medicine and Pharmaceutical Sciences, University of Douala, Douala, Cameroon.

Received: 12 February 2018 Accepted: 25 June 2018

Published online: 09 July 2018

References

1. Stanifer JW, Jing B, Tolan S, Helmke N, Mukerjee R, Naicker S, Patel U. The epidemiology of chronic kidney disease in sub-Saharan Africa: a systematic review and meta-analysis. Lancet Glob Health. 2014;2(3):e174-e181.

2. Naicker S. Burden of end-stage renal disease in sub-Saharan Africa. Clin Nephrol. 2010;74(Suppl 1):S13-6.

3. Wetmore JB, Collins AJ. Global challenges posed by the growth of endstage renal disease. Ren Replace Ther. 2016;2(1):15.

4. Liyanage T, Ninomiya T, Jha V, Neal B, Patrice HM, Okpechi I. Worldwide access to treatment for end-stage kidney disease: a systematic review. Lancet. 2015;385(9981):1975-82.

5. Kaze FF, Meto DT, Halle M-P, Ngogang J, Kengne A-P. Prevalence and determinants of chronic kidney disease in rural and urban Cameroonians: a cross-sectional study. BMC Nephrol. 2015;16(1):117.

6. Halle MP, Ashuntantang G, Kaze FF, Takongue C, Kengne A-P. Fatal outcomes among patients on maintenance haemodialysis in sub-Saharan Africa: a 10-year audit from the Douala general Hospital in Cameroon. BMC Nephrol. 2016:17(1):165.

7. Fouda H, Ashuntantang G, Kaze F, Halle M-P. Survival among chronic hemodialysed patient in Cameroon. Pan Afr Med J. 2017:26:97.

8. Perico N, Remuzzi G. Chronic kidney disease in sub-Saharan Africa: a public health priority. Lancet Glob Health. 2014;2(3):e124-e125.

9. Arogundade FA, Barsoum RS. CKD prevention in sub-Saharan Africa: a call for governmental, nongovernmental, and community support. Am J Kidney Dis. 2008:51(3):515-23.

10. Filiopoulos V, Hadjiyannakos D, Vlassopoulos D. New insights into uric acid effects on the progression and prognosis of chronic kidney disease. Ren Fail. 2012;34(4):510-20

11. Nakagawa $T$, Kang DH, Feig D, Sanchez-Lozada LG, Srinivas TR, Sautin Y, Ejaz $A A$, Segal M, Johnson RJ. Unearthing uric acid: an ancient factor with recently found significance in renal and cardiovascular disease. Kidney Int. 2006;69(10):1722-5.

12. Jalal DI, Chonchol M, Chen W, Targher G. Uric acid as a target of therapy in CKD. Am J Kidney Dis. 2013;61(1):134-46.

13. Tsai C-W, Lin S-Y, Kuo C-C, Huang C-C. Serum uric acid and progression of kidney disease: a longitudinal analysis and mini-review. PLoS One. 2017; 12(1):e0170393.

14. Chonchol M, Shlipak MG, Katz R, Sarnak MJ, Newman AB, Siscovick DS, Kestenbaum B, Carney JK, Fried LF. Relationship of uric acid with progression of kidney disease. Am J Kidney Dis. 2007:50(2):239-47.

15. Altemtam N, Russell J, El Nahas M. A study of the natural history of diabetic kidney disease (DKD). Nephrol Dial Transplant. 2012;27(5):1847-54.

16. Yen CJ, Chiang CK, Ho LC, Hsu SH, Hung KY, Wu KD, Tsai TJ. Hyperuricemia associated with rapid renal function decline in elderly Taiwanese subjects. J Formos Med Assoc. 2009:108(12):921-8.

17. Ficociello LH, Rosolowsky ET, Niewczas MA, Maselli NJ, Weinberg JM, Aschengrau A, Eckfeldt JH, Stanton RC, Galecki AT, Doria A, et al. High-normal serum uric acid increases risk of early progressive renal function loss in type 1 diabetes: results of a 6-year follow-up. Diabetes Care. 2010;33(6):1337-43.

18. Kuo CF, Luo SF, See LC, Ko YS, Chen YM, Hwang JS, Chou IJ, Chang HC, Chen HW, Yu KH. Hyperuricaemia and accelerated reduction in renal function. Scand J Rheumatol. 2011;40(2):116-21. 
19. Ishani A, Grandits GA, Grimm RH, Svendsen KH, Collins AJ, Prineas RJ, Neaton JD. Association of single measurements of dipstick proteinuria, estimated glomerular filtration rate, and hematocrit with 25-year incidence of end-stage renal disease in the multiple risk factor intervention trial. J Am Soc Nephrol. 2006;17(5):1444-52.

20. Hsu CY, Iribarren C, McCulloch CE, Darbinian J, Go AS. Risk factors for endstage renal disease: 25-year follow-up. Arch Intern Med. 2009;169(4):342-50.

21. Ben-Dov IZ, Kark JD. Serum uric acid is a GFR-independent long-term predictor of acute and chronic renal insufficiency: the Jerusalem lipid research clinic cohort study. Nephrol Dial Transplant. 2011;26(8):2558-66.

22. Iseki K, Ikemiya Y, Inoue T, Iseki C, Kinjo K, Takishita S. Significance of hyperuricemia as a risk factor for developing ESRD in a screened cohort. Am J Kidney Dis. 2004;44(4):642-50.

23. Kanbay M, Ozkara A, Selcoki Y, Isik B, Turgut F, Bavbek N, Uz E, Akcay A, Yigitoglu R, Covic A. Effect of treatment of hyperuricemia with allopurinol on blood pressure, creatinine clearence, and proteinuria in patients with normal renal functions. Int Urol Nephrol. 2007;39(4):1227-33.

24. Siu YP, Leung KT, Tong MK, Kwan TH. Use of allopurinol in slowing the progression of renal disease through its ability to lower serum uric acid level. Am J Kidney Dis. 2006;47(1):51-9.

25. Goicoechea M, de Vinuesa SG, Verdalles U, Ruiz-Caro C, Ampuero J, Rincon A Arroyo D, Luno J. Effect of allopurinol in chronic kidney disease progression and cardiovascular risk. Clin J Am Soc Nephrol. 2010;5(8):1388-93.

26. Adejumo O, Okaka E, Okwuonu C, Ojogwu L. Hyperuricemia in predialysis chronic kidney disease patients in Southern Nigeria. Sahel Med J. 2016;19(1):21-6.

27. Mahamat Abderraman G, Hamat I, Tondi Z, Lemrabott A, Faye M, Moustapha C, Sabi K, Ka K, Abdou N, Boucar D. Hyperuricemia in patients with chronic renal failure in the general hospital of national reference of N'Djamena (Chad). Open J Nephrol. 2017;7:9-18.

28. Edwards NL. The role of hyperuricemia and gout in kidney and cardiovascular disease. Cleve Clin J Med. 2008;75(Suppl 5):S13-6.

29. Stibưrková B, Pavlíková M, Sokolová J, Kožich V. Metabolic syndrome, alcohol consumption and genetic factors are associated with serum uric acid concentration. PLoS One. 2014;9(5):e97646.

30. de Oliveira EP, Burini RC. High plasma uric acid concentration: causes and consequences. Diabetol Metab Syndr. 2012;4(1):12.

31. Villegas R, Xiang Y-B, Cai Q, Fazio S, Linton M, Li H, Elasy T, Zheng W, Shu $X O$. Prevalence and determinants of hyperuricemia in middle-aged, urban Chinese men. Metab Syndr Relat Disord. 2010;8(3):263-70.

32. Chalmers J, MacMahon S, Mancia G, Whitworth J, Beilin L, Hansson L, Neal B, Rodgers A, Ni Mhurchu C, Clark T. 1999 World Health OrganizationInternational Society of Hypertension Guidelienes for the management of hypertension. Guidelines sub-committee of the World Health Organization. Clin Exp Hypertens. 1999;21(5-6):1009-60.

33. Zhao $Y$, Yang $X$, Lu W, Liao H, Liao F. Uricase based methods for determination of uric acid in serum. Microchim Acta. 2009;164:1):1-6.

34. Junge W, Wilke B, Halabi A, Jarausch J, Klein G. Reference intervals for total protein in collected and random urine using the Benzethonium chloride. Clin Chem. 2006;52:157.

35. Mazzachi BC, Peake MJ, Ehrhard V. Reference range and method comparison studies for enzymatic and Jaffé créatinin assays in plasma and serum, and early morning urine. Clin Lab. 2000:46:53-5.

36. Levey AS, Stevens LA, Schmid CH, Zhang YL, AFr C, Feldman HI, KJ W Eggers $P$, Van Lente $F$, Greene $T$, et al. A new equation to estimate gloomerular filtration rate. Ann Intern Med. 2009;150(9):604-12.

37. Stevens PE, Levin A. Evaluation and management of chronic kidney disease: synopsis of the kidney disease: improving global outcomes 2012 clinical practice guideline. Ann Intern Med. 2013;158(11):825-30.

38. Hochberg MC, Smolen JS, Weinblatt ME. Rheumatology. New York: Mosby; 2003

39. Keys A, Fidanza F, Karvonen MJ, Kimura N, Taylor HL. Indices of relative weight and obesity. Int J Epidemiol. 2014;43(3):655-65.

40. Patel KV. Epidemiology of anemia in older adults. Semin Hematol. 2008; 45(4):210-7

41. Third Report of the National Cholesterol Education Program (NCEP) Expert panel on detection, evaluation, and treatment of high blood cholesterol in adults (adult treatment panel III) final report. Circulation. 2002;106(25):3143-421.

42. Noone DG, Marks SD. Hyperuricemia is associated with hypertension, obesity, and albuminuria in children with chronic kidney disease. J Pediatr. 2013;162(1):128-32.
43. Krishnan E. Reduced glomerular function and prevalence of gout: NHANES 2009-10. PLoS One. 2012;7(11):e50046.

44. Kahn AM. Effect of diuretics on the renal handling of urate. Semin Nephrol. 1988:8(3):305-14.

45. Kanellis J, Watanabe S, Li JH, Kang DH, Li P, Nakagawa T, Wamsley A, Sheikh-Hamad D, Lan HY, Feng L, et al. Uric acid stimulates monocyte chemoattractant Protein-1 production in vascular smooth muscle cells via mitogen-activated protein kinase and Cyclooxygenase-2. Hypertension. 2003:41(6):1287-93.

46. Perlstein TS, Gumieniak O, Williams GH, Sparrow D, Vokonas PS, Gaziano M, Weiss ST, Litonjua AA. Uric Acid and the Development of Hypertension. The Normative Aging Study. Hypertension. 2006;48(6):1031-6.

47. Sedaghat S, Hoorn EJ, van Rooij FJA, Hofman A, Franco OH, Witteman JCM, Dehghan A. Serum uric acid and chronic kidney disease: the role of hypertension. PLoS One. 2013;8(11):e76827.

48. Reaven GM. The kidney: an unwilling accomplice in syndrome X. Am J Kidney Dis. 1997:30(6):928-31.

49. McAdams-DeMarco MA, Maynard JW, Coresh J, Baer AN. Anemia and the onset of gout in a population-based cohort of adults: atherosclerosis risk in communities study. Arthritis Res Ther. 2012;14(4):R193.

50. Bergamini C, Cicoira M, Rossi A, Vassanelli C. Oxidative stress and hyperuricaemia: pathophysiology, clinical relevance, and therapeutic implications in chronic heart failure. Eur J Heart Fail. 2009;11(5):444-52.

\section{Ready to submit your research? Choose BMC and benefit from:}

- fast, convenient online submission

- thorough peer review by experienced researchers in your field

- rapid publication on acceptance

- support for research data, including large and complex data types

- gold Open Access which fosters wider collaboration and increased citations

- maximum visibility for your research: over $100 \mathrm{M}$ website views per year

At BMC, research is always in progress.

Learn more biomedcentral.com/submissions 